\title{
Just Another Pelvic Mass?
}

\author{
Pablo Ruiz Sada ${ }^{1}$, Julia Ruiz Sada ${ }^{2}$, Javier Ángel López De la Osa ${ }^{3}$, Esther Niño Aragón ${ }^{4}$, Mikel Escalante Boleas ${ }^{5}$ \\ ${ }^{15}$ th year Resident, Internal Medicine, HCU Basurto, Bilbao, Spain \\ 23th year Resident, Obstetrics and Gynaecology, HU Miguel Servet, Zaragoza, Spain. \\ 35th year Resident, Internal Medicine, HCU Cruces, Barakaldo, Spain \\ 45th year Resident, Internal Medicine, Fundació Hospital de I'Esperit Sant, Santa Coloma de Gramenet, Spain \\ ${ }^{5} \mathrm{PhD}$ Consultant, Internal Medicine, HCU Basurto, Bilbao, Spain
}

Received: 06/08/2015

Accepted: 23/08/2015

Published: 09/09/2015

How to cite this article: Ruiz Sada P, Ruiz Sada J, López De la Osa JA, Niño AragónE, Escalante Boleas M. Just another pelvic mass? EJCRIM

2015;2:doi:10.12890/2015_000269

Conflicts of Interests: The authors declare that there are no competing interests.

Patient's Consent: The authors declare they obtained patient's consent for publication.

This article is licensed under a Commons Attribution Non-Commercial 4.0 License

\section{ABSTRACT}

Objectives: To describe the case of a patient presenting with weight loss and a pelvic mass.

Materials and methods: We performed an advanced search in MEDLINE using the key words "wasting syndrome", "actinomycosis" and "pelvic mass".

Results: A 63-year-old woman had a well-delimited and tender pelvic mass and was found to be anaemic. Before scans were performed, clinicians considered malignancy and other diagnoses.

Conclusion: Actinomycosis is a chronic infection that usually affects the abdomen. Because of the lack of specificity of clinical and radiological findings, a multidisciplinary approach is the keypoint for making an accurate diagnosis.

\section{LEARNING POINTS}

- Wasting syndromes not related to malignancy are rare but can mimic a typical paraneoplastic wasting syndrome.

- Although a pelvic mass is often due to gynaecological involvement, a broad differential diagnosis should first be considered.

- In contrast to the principle of Occam's razor, the simplest explanation is not always the correct one.

\section{KEYWORDS}

Wasting syndrome, actinomycosis, pelvic mass.

\section{INTRODUCTION}

Actinomycosis is a chronic infection caused by Actinomyces spp. that usually affects a single organ. Although abdominal involvement is described in medical records going back more than 150 years, diagnosis is still a challenge for the clinician. Indeed, the condition has been described as "the most misdiagnosed disease". This commensal bacillus causes such a non-specific range of clinical and radiological manifestations that a multidisciplinary and often a surgical approach is required. We describe the case of a woman complaining of weight loss and an abdominal mass.

\section{CASE REPORT}

A 63-year-old woman presented with a 1-month history of abdominal tenderness and a solid suprapubic mass. She complained of hyporexia, unexplained weight loss and 1 week of well-tolerated fever. She was allergic to iodine-based contrast, had well-controlled diabetes and a 
past history of operated bladder prolapse and appendectomy. She was Bolivian but denied recent travel to her home country.

On physical examination, there was no fever, heart murmurs or swollen lymph nodes, and a solid, well-delimited and tender mass was evident on palpation. Laboratory findings revealed a C-reactive protein (CRP) of $6.43 \mathrm{mg} / \mathrm{dl}$ (normal range 0-0.50), erythrocyte sedimentation rate (ESR) of $100 \mathrm{~mm} / \mathrm{h}$ and mild normocytic anaemia (Hb $101 \mathrm{~g} / \mathrm{l}$, mean cell volume (MCV) 86.5). Simple x-rays were normal and an abdominal CT scan showed inflammatory involvement of the prepubic fat extending to the genital area with diffuse inflammatory pelvic changes in front of the iliac vessels and surrounding the fat bladder dome; no gynaecological or gastrointestinal involved was indicated (Fig. 1). Echoguided biopsy was performed and the pathologist reported the presence of fibroadipose tissue and acute abscess inflammation.

A gynaecologist ruled out any gynaecological condition as did the surgical team who removed the mass. Intraoperative biopsies showed the presence of fistula and an abscess containing basophils and filaments suggesting actinomyces (Figs. 2 and 3). Full dose antibiotic treatment was given with 5 million units of intravenous penicillin G every $6 \mathrm{~h}$ for 4 weeks followed by oral amoxicillin 500 mg three times daily for 1 year. A CT control scan showed progressive improvement with full resolution of the infection.
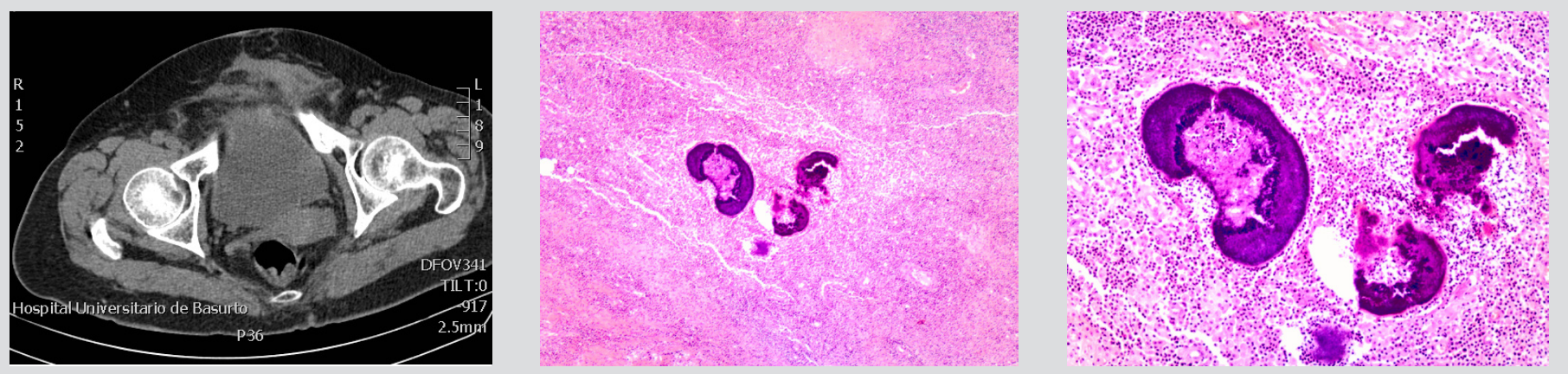

Figure 1: A diffuse inflammatory pelvic tumour in front of the iliac vessels and surrounding the fat bladder dome and extending up to L3-L4.

Figure 2: Abscess containing basophils and filaments suggesting actinomyces.

Figure 3: Filaments and sulfur granules.

\section{DISCUSSION}

Actinomyces is a rare filamentous, gram positive and anaerobic bacterium that causes a chronic and slowly progressive granulomatous disease. It usually remains confined to a specific area, most frequently the cervicofacial region (50\%), followed by thoracic and abdominal involvement, which each account for $20 \%$ of cases $^{[1]}$. Although it is a commensal inhabitant of the oral cavity and urogenital tract, it can invade necrotic tissue following a previous mucosal injury and cause polymicrobial infection. Thus, it is usually triggered after abdominal surgery (both appendectomy and cholecystectomy), trauma (a fish bone injuring the digestive tract), neoplasms or intrauterine contraceptive device (IUCD) use. However, is 2-4 times more common in men, mainly affects middle-aged individuals and has a worldwide distribution, without a race or social class preference ${ }^{[2]}$.

This pathogen characterized by slow growth tends to form masses and causes localized infection. Therefore, clinical manifestations are few and non-specific, ranging from malaise, chills and fever to a palpable mass and weight loss. Laboratory results and radiological findings also show a lack of specificity ${ }^{[3]}$. These difficulties hinder the differential diagnosis with malignancy, a condition which must be recognised by clinicians. Blood tests show an inflammatory picture that can be compatible with neoplasm. Ultrasound is the scan of choice following the finding of an abdominal mass, but again, it might show features of either an abscess or a solid tumour. However, a CT scan allows the clinician to determine the location and extent of disease, but does not rule out malignancy either ${ }^{[4]}$. The definitive diagnostic test is usually based on histology (the identification of actinomycosis sulfur granules) or microbiology (culturing the organism). According to some sources, less than $10 \%$ of cases are diagnosed preoperatively, so there is a need for a high level of suspicion and collaboration between specialties ${ }^{[1]}$.

Although we were on the brink of sending the patient for gynaecological oncology investigation, we first referred her to a general gynaecologist. The patient had tumour markers within normal limits. The most common ovarian tumours in postmenopausal women are carcinomas of epithelial origin which often have elevated tumour markers (serous and mucinous subtypes account for more than $90 \%$ of such cancers). Although they are not currently used for diagnosis, CA125 is high in more than 75-80\% of patients with serous ovarian carcinoma, while CA19.9 is specific for mucinous carcinoma. Transvaginal ultrasound visualization of a normal uterus and appendages is used to support or refute the diagnosis and in our case helped us to exclude initially suspected conditions ${ }^{[5]}$. 


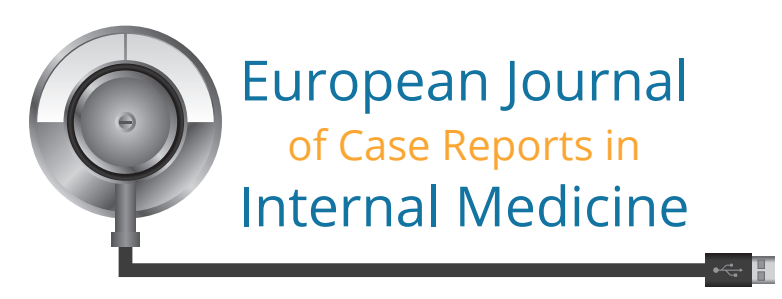

Penicillin is the preferred drug of choice, and is given as 2-5 million units four times intravenously per day for the first 6 weeks followed by oral penicillin or amoxicillin for 6-12 months. Patients allergic to penicillin should be treated with tetracycline, erythromycin or clindamycin. Surgery plays a role in large, persistent and recurrent abscesses or when a neoplasm cannot be ruled out. Mortality is rare and the outcome is favourable in more than $90 \%$ of cases with both medical and surgical treatment ${ }^{[2]}$.

\section{REFERENCES}

1. Garner JP, Macdonald M, Kumar PK. Abdominal actinomycosis. Int J Surg 2007:5: 441-448.

2. Wong VK, Turmezei TD, Weston VC. Actinomycosis. BMJ 2011;343:d6099

3. Galata CL, Vogelmann R, Gaiser T, Post S, Horisberger K. Abdominopelvic actinomycosis in three different locations with invasion of the abdominal wall and ureteric obstruction: An uncommon presentation. Int J Surg Case Rep 2015;12:48-51.

4. Zamani F, Sohrabi M. Clinical, endoscopic, and histopathological aspects of sigmoid actinomycosis; a case report and literature review. Middle East J Dig Dis 2015;7:41-44.

5. Pavlik EJ, Ueland FR, Miller RW, et al. Frequency and disposition of ovarian abnormalities followed with serial transvaginal ultrasonography. Obstet Gynecol 2013;122:210. 